\title{
EIN VERLAG \\ UND SEINE \\ GESCHICHTE
}




\title{
EIN VERLAG UND SEINE GESCHICHTE \\ DREIHUNDERT JAHRE J. B. METZLER STUTTGART
}

\author{
J. B. METZLERSCHE \\ VERLAGSBUCHHANDLUNG \\ STUTTGART
}


CIP-Kurztitelaufnahme der Deutschen Bibliothek

Wittmann, Reinhard:

Ein Verlag und seine Geschichte:

300 Jahre J. B. Metzler Stuttgart /

Reinhard Wittmann.

- Stuttgart: Metzler, 1982.

ISBN 978-3-476-00481-9

NE: J. B. Metzlersche Verlagsbuchhandlung

und Carl Ernst Poeschel Verlag 〈Stuttgart〉

ISBN 978-3-476-00481-9

ISBN 978-3-476-03157-0 (eBook)

DOI 10.1007/978-3-476-03157-0

(C) Springer-Verlag GmbH Deutschland 1982

Ursprünglich erschienen bei J. B. Metzlersche Verlagsbuchhandlung und Carl Ernst Poeschel Verlag GmbH in Stuttgart 1982 


\section{INHALT}

\section{Vorwort}

Die Anfänge 1670-1716:

Johann Gottfried Zubrod und Augustus Metzler

15 Stuttgarts Buchgewerbe bis 1670

27 Der erste Buchhändler: J. G. Zubrod

38 Die Entwicklung der Firma 1670-1690

60 Der "Gründer «: Augustus Metzler

81 Der Verlag und sein Programm 1690-1713

105 Intermezzo: Christoph Erhard 1714-1716

Johann Benedikt Metzler der Ältere

und die Firma von 1716-1753

111 Die Etablierung

135 Die Sozietät der Rivalen

148 Trennung und letzte Jahre

Von Bengel bis Lessing: Der Verlag und seine Autoren

174 Die Schwerpunkte

192 Johann Albrecht Bengel

204 Johann Jakob Moser

213 Gotthold Ephraim Lessing

Die Firma 1754-1795: J. B. Metzler d.J.

223 Der Buchmarkt in der Krise: Nachdruck und Nettohandel 253 Württembergs literarisches Leben und die Carlsschule 
Von Hiller bis Schiller: Der Verlag und seine Autoren 263 Die Sachgebiete 294 Schubart als Verlagsautor 300 Schiller und Metzler

Interregnum 1795-1815: Christoph Heinrich Erhard 315 Die Übernahme der Firma

330 Politik und Buchmarkt in Württemberg 1795-1815

338 Stuttgarts Buchhandel im Umbruch

Die Firma 1816-1867: Heinrich Erhard

357 Stuttgarts Buchwesen zwischen Restauration und Gründerzeit

365 Der Weg eines liberalen Verlegers

Von Voss bis C. F. Meyer:

Der Verlag im 19. Jahrhundert

381 Die Schwerpunkte

414 Zeitschriften und Zeitungen

435 Die Germania

443 Die "großen" Autoren:

443 Johann Heinrich Voss

457 Joseph Görres

478 Wilhelm Hauff

482 Charles Sealsfield

500 Josef Viktor Scheffel

516 Conrad Ferdinand Meyer

520 Versäumte Gelegenheiten

Stagnation: Die Familie Werlitz 1870-1907

543 Teilhaber und Widersacher:

Leopold Werlitz und Adolf Bonz

554 Egon und Arthur Werlitz

561 Der Verlag am Ende des 19. Jahrhunderts 
Der Verlag 1908-1945: Alfred Druckenmüller

565 Die Entwicklung bis 1933

578 Dichtung und Volkstum - der Verlag im Dritten Reich

Die Entwicklung von 1945 bis zur Gegenwart

591 Der Neubeginn nach Kriegsende 1945

605 Hermann Leins als Verleger

610 Die fünfziger Jahre - ein Programm der klaren Linien

612 Fachspezifische Orientierung des Verlagsprogramms die sechziger Jahre

618 Die siebziger und frühen achtziger Jahre - ein Ausblick

625 Anmerkungen

671 Quellen- und Literaturverzeichnis

681 Bibliographie des Metzler-Verlages von 1682 bis 1848

777 Register

795 Bildquellen 


\section{VORWORT}

Eine Verlagsgeschichte, die mehr sein will als eine jener mehr oder minder aufwendigen Festschriften, in denen sich ein traditionsreiches Haus mit stolzem Behagen feiern läßt, steht vor mannigfachen Problemen. Denn ihr Erkenntnisinteresse ist über die Firmenhistorie hinaus darauf gerichtet, zu einer Sozialgeschichte der literarischen Vermittlung beizutragen - zu untersuchen also, wie im Laufe der Jahrhunderte unter wechselnden kulturellen, politischen, sozialen und wirtschaftlichen Bedingungen die kommerzielle Herstellung und Verbreitung von Geistesgut aussah, aber ebenso, wie dieser Vermittlungsprozeß seinerseits einwirkte auf die Produktion und Rezeption von Büchern. Dabei gilt es, allen Bereichen dieses Vermittlungsprozesses gleichermaßen Aufmerksamkeit zu widmen, von den ersten Autorenkontakten bis zum Verkauf der Neuerscheinung über den Ladentisch. In die Darstellung sollten aber nicht nur Autoren und Leser als Komponenten des literarischen Lebens einbezogen werden, sondern gleichermaßen die äußeren Voraussetzungen, unter denen das Kommunikationssystem Buchhandel überhaupt erst existieren und funktionieren kann - eben jener genannte Rahmen aus lokalen und regionalen, religiösen, geistigen, gesellschaftlichen, politischen, ökonomischen und natürlich buchhandelsgeschichtlichen Entwicklungen und Einflüssen.

Solchen Idealvorstellungen zu genügen, wird jedoch dieser Darstellung nur in bescheidenem Maße gelingen - auch wenn sie dickleibig genug ausgefallen, mit Anmerkungshalden, Verlagsbibliographie und Registern versehen ist. Nicht ohne Grund gibt es bisher nur eine Handvoll wissenschaftlich zureichender Verlagsmonographien, die einen größeren Zeitraum, gar einige Jahrhunderte umspannen. Erhebliche 
Schwierigkeiten mit den Quellen wie mit der Methode reduzieren so manches hochgespannte Projekt auf ein bescheidenes Maß. Im bisher kaum erforschten Bereich der Verlagsgeschichte ist wissenschaftlicher Fortschritt noch immer fast ausschließlich durch Kenntniserweiterung, durch Aufspüren und Einordnen neuer Quellen zu erreichen. Für ein Forschen auf höherer Reflexionsebene, für souveräne vergleichende Zusammenschau auf solidem Materialfundament fehlen weitgehend die Voraussetzungen, will man sich nicht vorschnell auf spekulatives Gebiet begeben. Für eine gerechtere Beurteilung dessen, was diese Darstellung erreichen konnte und was man vergeblich darin suchen wird, muß deshalb von solchen Problemen die Rede sein.

Eine Fundgrube wie das Archiv des Cotta-Verlages, das Generationen von Dissertanden ins Brot setzen könnte, gibt es für keinen zweiten Verlag. Die umständliche, oft fruchtlose Suche nach verstreuten Quellenresten - sämtliche Archivalien, Korrespondenzen, Bestände des J. B. Metzler-Verlags sind mit dem Verlagshaus durch einen Bombenangriff am 7./8. Oktober 1943 zerstört worden - bildete einen erheblichen Teil der Arbeit an diesem Buch, ohne daß es mit all dem zusammengetragenen Material überfrachtet werden durfte. Obgleich gerade die Buchhandelsgeschichte dringend der Überprüfung längst etablierter Thesen durch neue Quellenerschließung bedarf, können solche Dokumente doch nicht isoliert gesehen und, so verführerisch das sein mag, überinterpretiert werden, solange nicht genügend gesichertes Vergleichsmaterial vorliegt. Deshalb hat auch diese Verlagsgeschichte sich stärker als beabsichtigt bei der Deutung der mitgeteilten Quellen zurückgehalten. Bei deren Darbietung selbst wurde dagegen eher großzügig verfahren. Manches mag etwas breit zitiert erscheinen, kann aber gerade angesichts der dürftigen Quellensituation der Buchgeschichte für das 17. und frühe 18. Jahrhundert künftiger Forschung nützlich sein.

Obgleich das Buch doppelt so umfangreich geworden ist wie geplant, hat es sein ursprüngliches Ziel nur teilweise erreicht. Der naive Plan etwa, sämtliche noch auffindbaren Verlagsveröffentlichungen einzusehen, scheiterte schnell an den Realitäten des bibliothekarischen Leihverkehrs. Manches aufschlußreiche Buch mag mir deshalb entgangen sein und nur 
in der Bibliographie auftauchen, obgleich es eigener Erwähnung wert gewesen wäre. Die seitenlangen Titellisten, die so manche Verlagshistorie füllen, wurden bewußt vermieden. Dafür ist die Bibliographie im Anhang auf Vollständigkeit bedacht, auch wenn dies als kaum realisierbares Unterfangen gerade für die Anfangsjahrzehnte der Firmengeschichte erscheint. Bei allen unvermeidlichen Lücken mag dieser meines Wissens erste Versuch einer Gesamtbibliographie eines Verlages vom 17. bis zur Mitte des 19. Jahrhunderts doch vielleicht den Anstoß für dringend benötigte Verzeichnisse ähnlicher Art geben (etwa für Cotta).

Eine lückenlose Durchforschung der ergiebigsten Quelle für die deutsche Buchhandelsgeschichte seit 1834 war nicht möglich: von den Hunderttausenden engbedruckter Quartseiten des Börsenblatts für den Deutschen Buchhandel konnten nur jene bis etwa 1870 ausgiebig benutzt werden. Ein Exemplar der fürs gesamte 19. Jahrhundert überaus aufschlußreichen Süddeutschen Buchhändlerzeitung war mir nicht zugänglich.

Die ebenfalls geplante breite Berücksichtigung des geistigen und sozialen Umfeldes dieser dreihundert Jahre in Württemberg ließ sich nicht verwirklichen - sie hätte die Darstellung völlig gesprengt. Deshalb finden die Perspektiven der württembergischen Geistes- und Sozialgeschichte, des Pietismus, der Zensur, des Lesepublikums usw. meist nur in Andeutungen und Verweisen Erwähnung.

In erfreulichem Maße (wenn auch nur für bestimmte Perioden und Bereiche) kamen jedoch unbekannte Quellen in öffentlichem und privatem Besitz zutage - dank der "schwäbischen Schwäche für umfangreiches Schreibwerk " (Dieter Narr). Die ergiebigen Bestände im Hauptstaatsarchiv Stuttgart zum württembergischen Buchhandel des 17. bis 19. Jahrhunderts sind bisher fast unausgewertet geblieben, darunter wichtige Materialien zur Verlagshistorie. In bescheidenerem Maße enthalten auch das Staatsarchiv Ludwigsburg und das Archiv der Evangelischen Landeskirche Stuttgart (das die Akten des ehemaligen Konsistoriums bewahrt) aufschlußreiche Dokumente; Briefe Heinrich Erhards an den Herausgeber der Germania, Franz Pfeiffer, befinden sich in der Württembergischen Landesbibliothek, eine Anzahl von Einzelbriefen Erhards an schwäbische Geistesgrößen hat das Schiller-Nationalmuseum gesammelt. Wichtige 
Familiendokumente fanden sich in Wuppertaler Privatbesitz. Schließlich erwies sich der mangelnde Geschichtssinn bzw. die Freigiebigkeit der ehemaligen Verlagsinhaber am Ende des 19. Jahrhunderts, Egon und Arthur Werlitz, als besonderer Glücksfall. Sie haben die Zimelien des damals noch reichbestückten Firmenarchivs (ein Teil war bei der Trennung der Erhard-Schwiegersöhne makuliert worden) dem Autographensammler Rudolf Kauffmann überlassen - vom frühesten Originalprivileg J. G. Zubrods bis zum Kärtchen der Ottilie Wildermuth sind rund 150 Stücke auf diese Weise der Vernichtung entgangen und nun im Stadtarchiv Stuttgart verwahrt.

Die Darstellung hatte sich nach den Quellen zu richten, was einen gewissen Methodenpluralismus und Wechsel der Perspektiven bedingte: In manchen Jahrzehnten steht die Geistesgeschichte im Vordergrund, in anderen die buchhändlerische oder gar ınur` die Persönlichkeit eines Firmeninhabers. Manche Perioden werden vergleichsweise breit behandelt, andere dagegen kommen eher zu kurz - besonders die Zeit seit Beginn unseres Jahrhunderts. Doch liegt dies auch an einem dem Buchhistoriker wohlbekannten Phänomen: Unterlagen der jüngeren Vergangenheit werden häufig als nicht archivierungswürdig vernichtet. In diesem Fall trug vor allem die Zerstörung des Verlags im Zweiten Weltkrieg dazu bei, daß seine zweifellos besonders interessante Geschichte in den Zwanzigerjahren und im Dritten Reich entsprechend der dürftigen Materiallage relativ oberflächlich behandelt werden mußte. Generell hat sich im Fortschreiten der Darstellung die Perspektive verengt: Während etwa der Geistesgeschichte Württembergs im 18. Jahrhundert stärkere Aufmerksamkeit gewidmet wurde, schien eine vergleichbare Ausführlichkeit bei den politischen und geistigen Entwicklungen des 20. Jahrhunderts entbehrlich. Das letzte Kapitel, die Entwicklung des Verlags seit 1945, wurde von Bernd Lutz geschrieben, weil eine "historische" Darstellung hier weniger sinnvoll erschien.

Auch wenn diese Verlagsgeschichte nicht allen eingangs genannten Forderungen zu genügen vermag, so kann sie doch mit einer nicht geringen Zahl neuer Dokumente und Quellen aufwarten - etwa zu den Schwabenvätern Bengel und J.J. Moser, zu Schiller und Voß, Görres 
und Börne, Friedrich List und Scheffel, aber auch zum wenig erforschten buchhändlerischen Alltag des 17. bis 19. Jahrhunderts. Bis auf die Briefe Mörikes und Sealsfields sowie einzelne Zitate sind alle wiedergegebenen Dokumente hier erstmals gedruckt. Es versteht sich, daß sämtliche Quellen in ihrer ursprünglichen Gestalt belassen wurden; Kürzungen sind vermerkt, ausgenommen bei den obligaten Eingangs- und Schlußformeln der Briefe und Eingaben. Die im Text oft abgekürzt zitierten Titel sind in der Bibliographie vollständig wiedergegeben.

Schließlich bleibt all denen zu danken, die über Jahre hinweg das Entstehen dieses Buches gefördert haben: den Archiven und Bibliotheken (besonders Friedrich Pfäfflin vom Deutschen Literaturarchiv Marbach) sowie den privaten Besitzern, die die Erlaubnis zur Veröffentlichung ihrer Bestände gaben. Die Bibliographie konnte auf Vorarbeiten von Ernst Metelmann zurückgreifen und wurde zunächst von Dr. Hiltrud Häntzschel, dann von Myriam Nothacker betreut. Ernst Metelmann hat genealogische und firmengeschichtliche Materialien bereitwillig zur Verfügung gestellt und manche Einzelhinweise gegeben, ebenso Herbert G. Göpfert.

Natürlich hat der J. B. Metzler-Verlag selbst am Entstehen dieses Buches in allen Stadien stets Anteil genommen; insbesondere Dr. Bernd Lutz hat mannigfache Unterstützung gewährt. Vor allem aber muß ich an dieser Stelle meiner Frau danken. Ohne ihre Geduld und Mithilfe wäre eine solche jahrelange Wochenendarbeit ohne universitäre oder sonstige Hilfskräfte unmöglich gewesen.

R. W. 